\title{
Analysis of Coil Misalignment in MCRWPT System
}

\author{
Junfeng Yanga, Xiaodong Zhang ${ }^{\mathrm{b}}$, Dandan Liu ${ }^{\mathrm{c}}$ \\ School of Electrical Engineering, Beijing Jiaotong University, Beijing 100044, China. \\ ayangjunfeng@bjtu.edu.cn, bxdzhang@bjtu.edu.cn, c15121435@bjtu.edu.cn
}

Keywords: Magnetic coupling resonant, wireless power transfer, mutual inductance, power and efficiency.

\begin{abstract}
Coil misalignment often takes place in wireless power transfer (WPT) systems. It may result in decrease of transfer efficiency and system instability. In this paper the mutual inductance model and the circuit equation of Magnetic Coupling Resonant WPT (MCRWPT) are established firstly. And the influence of the mutual inductance on the transfer characteristic parameters is analyzed. Then the transfer power and efficiency under three transfer modes with different sizes between two coils in the offset state are studied. Based on the analysis results, a method to improve the transfer efficiency of the MCRWPT system is proposed. Finally, the analysis results and the feasibility of the proposed method are verified, through the simulations and experiments.
\end{abstract}

\section{Introduction}

Wireless power transfer has been developed as a new technology for electric power transmission in recent years. Because it has many advantages, such as its superiority on convenience of being cordless, safety during high-power charging, ability to work in wet and harsh environment. One of the major means is magnetic coupling resonant technology, with time-varying electromagnetic field as a medium. It uses two or more with the same resonant frequency and high-quality factor of the electromagnetic resonance system to achieve wireless power transfer [1]. In the wireless power transfer system, the structure and parameters design of the transmitting and the receiving coils are very important to improve the power and efficiency. Generally, the two coils need to be well aligned to make the system work in its best state. However, in practice, misalignment between them often takes place, which will affect the coil coupling mutual inductance and then affect the power and efficiency [2]. Some scholars have studied the factors of mutual inductance [3] but mainly of them are focused on the analysis of mutual inductance of the two coaxial coils.

Based on this, the coupling effect of two parallel coils with different coil radius, axial offset and radial offset on mutual inductance are studied. The transfer power and efficiency under three typical transfer modes with different sizes between two coils in the offset state are analyzed. In order to reduce the influence of offset state on transfer efficiency, a method is put forward in this paper to increase the mutual inductance of the coil by changing the size of the receiving coil, and further to improve the transfer efficiency. A large number of simulations and experiments are carried out to verify the analysis results and the feasibility of the proposed method.

\section{Magnetic Coupling Transfer System Model}

Topology of MCRWPT system in Fig. 1 can be modeled by mutual inductance circuit theory [4,5]. It takes all parameters of resonance frequency, mutual inductance and internal resistance into account. The system usually consists of a high frequency power supply, transmitting and the receiving coils, a load resistance. RP (RS), LP (LS) and CP (CS) are the transmitting (receiving) side coil resistance, inductance and series compensation capacitor, RL is load resistance, $\mathrm{M}$ is the mutual inductance between two coils. 


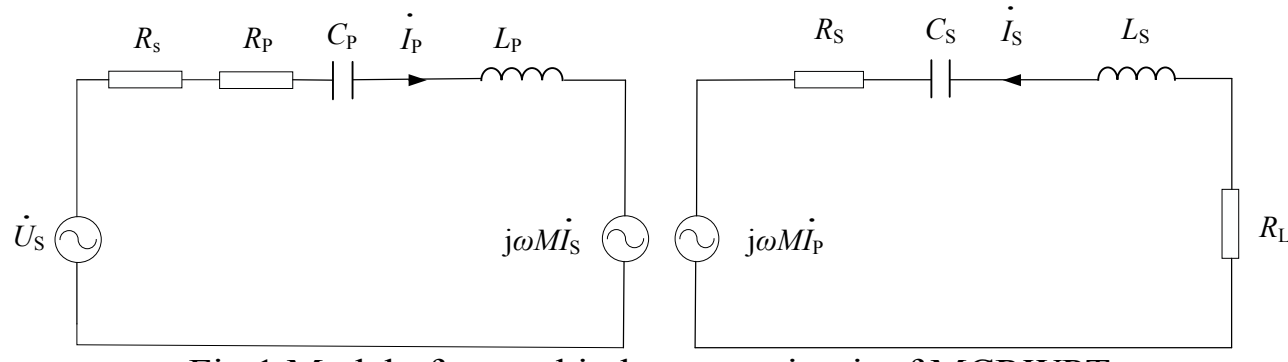

Fig.1 Model of mutual inductance circuit of MCRWPT

The equivalent circuit in primary side is demonstrated in Fig.2. Among it, $\mathrm{Zr}$ is the equivalent impedance transited from receiving side to the transmitting side. ZS is the impedance of receiving (secondary) side. ZP is the integral equivalent impedance of the transmitting(primary) side of the MCRWPT system.

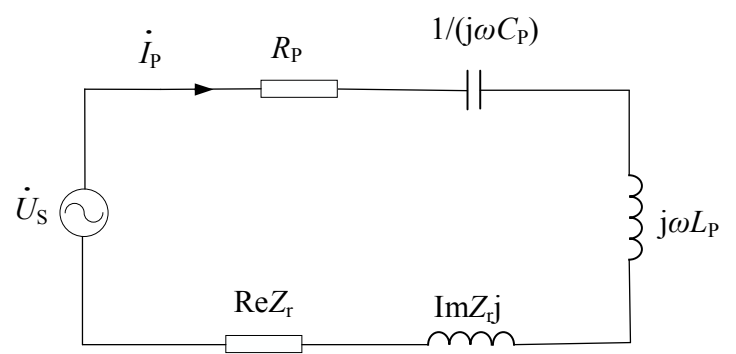

Fig.2 System equivalent circuit of MCR in primary side

$$
\begin{gathered}
Z_{\mathrm{r}}=\frac{\omega^{2} M^{2}}{Z_{\mathrm{S}}} \\
Z_{\mathrm{S}}=R_{\mathrm{S}}+R_{\mathrm{L}}+\mathrm{j} \omega L_{\mathrm{S}}-\frac{\mathrm{j}}{\omega C_{\mathrm{S}}} \\
Z_{\mathrm{p}}=R_{\mathrm{P}}+\operatorname{Re} Z_{\mathrm{r}}+\mathrm{j}\left(\omega L_{\mathrm{p}}-\frac{1}{\omega C_{\mathrm{p}}}+\operatorname{Im} Z_{\mathrm{r}}\right)
\end{gathered}
$$

Then the system input and output power when the system in the resonant state can be calculated as follow:

$$
\begin{gathered}
Z_{\mathrm{p}}=R_{\mathrm{P}}+\frac{\omega^{2} M^{2}}{\left(R_{\mathrm{S}}+R_{\mathrm{L}}\right)} \\
P_{\text {in }}=\operatorname{Re}\left(\dot{U}_{\mathrm{S}} \dot{I}_{\mathrm{P}}^{*}\right)=\frac{\left(R_{\mathrm{S}}+R_{\mathrm{L}}\right)\left|\dot{U}_{\mathrm{S}}\right|^{2}}{R_{\mathrm{P}}\left(R_{\mathrm{S}}+R_{\mathrm{L}}\right)+(\omega M)^{2}} \\
P_{\mathrm{L}}=\left|\dot{I}_{\mathrm{S}}\right|^{2} R_{\mathrm{L}}=\frac{\omega^{2} M^{2}\left|\dot{U}_{\mathrm{S}}\right|^{2} R_{\mathrm{L}}}{\left[R_{\mathrm{P}}\left(R_{\mathrm{S}}+R_{\mathrm{L}}\right)+(\omega M)^{2}\right]^{2}}
\end{gathered}
$$

And the transfer efficiency of the system is

$$
\eta=\frac{P_{\mathrm{L}}}{P_{\text {in }}}=\frac{\omega^{2} R_{\mathrm{L}} M^{2}}{\left(R_{\mathrm{P}}\left(R_{\mathrm{S}}+R_{\mathrm{L}}\right)+(\omega M)^{2}\right)\left(R_{\mathrm{S}}+R_{\mathrm{L}}\right)}
$$

In a specific MCR system, $\dot{U}_{\mathrm{S}}, \omega, R_{\mathrm{S}}, R_{\mathrm{a}}$ and $R_{\mathrm{L}}$ are given, so the change of mutual inductance will affects $P_{\text {in }}, P_{\mathrm{L}}$ and $\eta$ directly as follow:

$$
\frac{\partial P_{\text {in }}}{\partial M}=-2 \omega^{2}\left|\dot{U}_{\mathrm{S}}\right|^{2} \frac{\left(R_{\mathrm{S}}+R_{\mathrm{L}}\right) M}{\left(R_{\mathrm{P}}\left(R_{\mathrm{S}}+R_{\mathrm{L}}\right)+(\omega M)^{2}\right)^{2}}
$$




$$
\begin{gathered}
\frac{\partial P_{\mathrm{L}}}{\partial M}=2 \omega^{2}\left|\dot{U}_{\mathrm{S}}\right|^{2} R_{\mathrm{L}} \frac{M\left(R_{\mathrm{P}}\left(R_{\mathrm{S}}+R_{\mathrm{L}}\right)-\omega^{2} M^{2}\right)}{\left(R_{\mathrm{P}}\left(R_{\mathrm{S}}+R_{\mathrm{L}}\right)+(\omega M)^{2}\right)^{3}} \\
\frac{\partial \eta}{\partial M}=\frac{2 \omega^{2} R_{\mathrm{L}} R_{\mathrm{P}} M}{\left(R_{\mathrm{P}}\left(R_{\mathrm{S}}+R_{\mathrm{L}}\right)+(\omega M)^{2}\right)^{2}}
\end{gathered}
$$

Where the derivative of Pin to M (8) shows that Pin decreases monotonically with the increase of $\mathrm{M}$, and the derivative of $P_{\mathrm{L}}$ to $\mathrm{M}$ (9) shows that there is a maximum value of $P_{\mathrm{L}}$ at $M=\frac{\sqrt{R_{\mathrm{P}}\left(R_{\mathrm{S}}+R_{\mathrm{L}}\right)}}{\omega}$ point, $P_{\mathrm{L}}$ increases with the increase of $\mathrm{M}$ when less than $\mathrm{M}$, and decreases with the increase of $M$ when greater than $M$. The result of the derivative of $\eta$ to $M$ (10) shows that $\eta$ increases monotonically with the increase of $\mathrm{M}$.

Therefore, mutual inductance is the bridge of the coupling relationship between the two coils. With the offset of the coil, the mutual inductance between the coils would change, and the other transmission characteristics can be analyzed simultaneously.

\section{Analysis of Mutual Inductance Under Misalignment of Different Size Coils}

Coil misalignment will cause significant impact on the transfer efficiency and instability of MCRWPT system. Misalignment of different shapes of two coils have be studied [6], and the mutual inductance of two circular coils is larger and more effective. The angle offset under 10 degrees has little influence on the mutual inductance between the coils. Therefore, the system transmission characteristics of two circular parallel coils in the offset state are studied below.

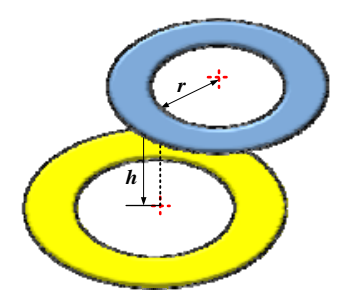

a) TL-RS

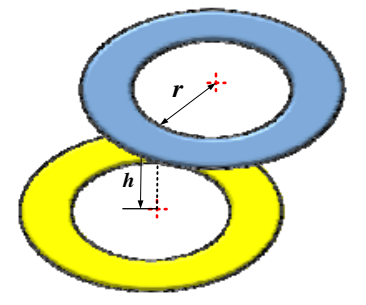

b) $\mathrm{T}=\mathrm{R}$

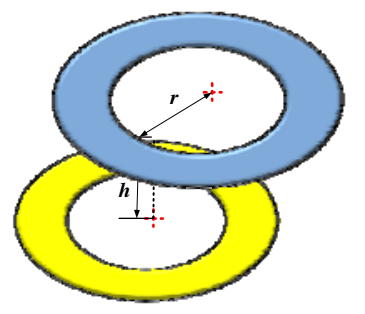

c) TS-RL

Fig. 3 The schematic diagram of parallel coil migration in three transfer modes

The energy transfer models between the coils are divided into three categories according to the size of the receiving coil: TL-RS, T=R and TS-RL, as shown in Fig.3. The yellow coil at the bottom represents the transmitting coil, with fixed radius and the one on the top represents receiving coil, with different radius. Curves of mutual inductance in three models following with radial offset and axial offset are shown as Fig. 4.

It can be seen from Fig. 4 that the mutual inductance of the two coils decreases monotonically with the growing of the axial offset. When the radius of the two coils is equal, the mutual inductance is the largest when there is no deviation. but the mutual inductance drops rapidly with the growing of axial and radial offset, which stabilizes after decay to a certain extent. The radius of the two coils is not equal, the mutual inductance between them have a great value when the axial offset is 0 and the radial offset is $\left|r_{1}-r_{2}\right|$. But when the offsets are the same, the mutual inductance of TS-RL model is larger than TL-RS's. This provides a theoretical reference for the selection of coil size and the placement spatial location. 


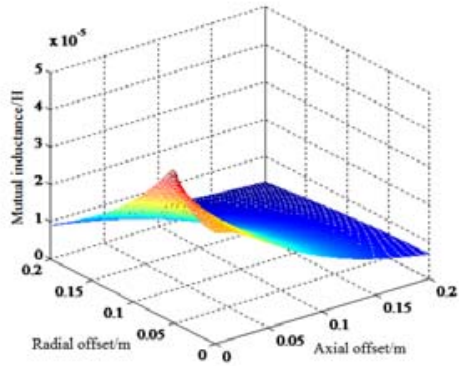

a) TL-RS

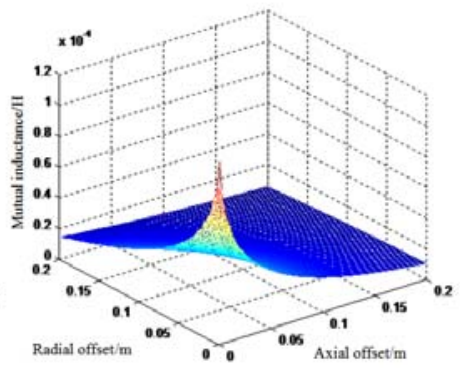

b) $\mathrm{T}=\mathrm{R}$

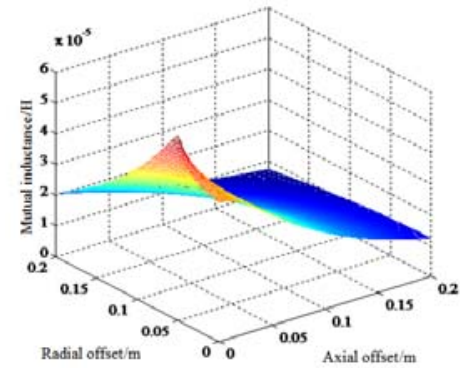

c) TS-RL

Fig.4 Change of mutual inductance in parallel coils deviation in three transfer modes

\section{Analysis of the Effect of Coil Misalignment on the Parameters of MCRWPT System}

Based on the above, the changing rule of mutual inductance in the three transmission modes under the axial and radial offset is obtained, and then the change of other transmission parameters under offset states can be analyzed through the mutual inductance change. By using the finite element software COMSOL, the simplified models with the same parameters as the actual coils in three transfer modes is established, and the lumped parameters of the different coils are obtained. The coils' parameters are listed in Tab.1. Changes of input power, transfer power and efficiency in three models are shown in Fig.5.

Table 1. the Parameters of Coils

\begin{tabular}{cccc}
\hline Models & TL-RS & T=R & TS-RL \\
\hline the radius of transmit coil & $20 \mathrm{~cm}$ & $20 \mathrm{~cm}$ & $20 \mathrm{~cm}$ \\
the radius of receiving coil & $15 \mathrm{~cm}$ & $20 \mathrm{~cm}$ & $25 \mathrm{~cm}$ \\
turns of transmit coil & 10 & 10 & 10 \\
turns of transmit coil & 10 & 10 & 10 \\
\hline
\end{tabular}

The first column in Fig.5 demonstrates the relationship between the input power and offsets under the constant-voltage source. Pin increases monotonically with the increase of radial offset and axial offset. Under the same offsets, the maximum input power comes from TL-RS model, and the minimum from TS-RL model. Higher input power gives rise to lower system transfer efficiency, therefore the TL-RS model does not suitable for the MCRWPT system.

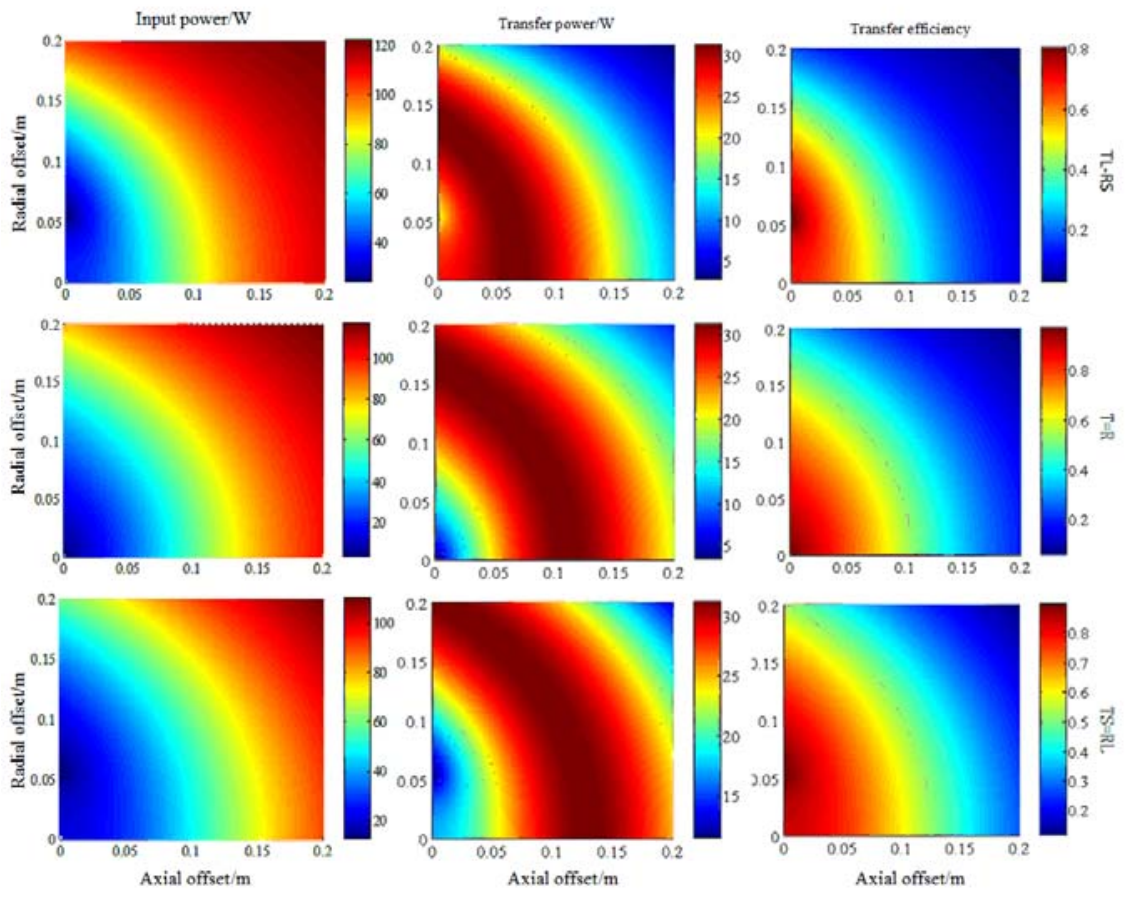

Fig.5 Input power, transfer power and efficiency vary with offsets in three transfer modes 
According to the analysis of the second section, the change of mutual inductance will directly affect the transfer power of the system, and there is an optimal mutual inductance, which makes the transfer power largest. Moreover, the efficiency is positively related to the mutual inductance. The second and third column in Fig.5 show the changes of transfer power and efficiency with axial and radial offsets in three modes.

In the TL-RS mode, the maximum load efficiency occurs at the axial distance of $0 \mathrm{~cm}$ and the radial distance at $5 \mathrm{~cm}$. At the axial offset $5 \mathrm{~cm}-10 \mathrm{~cm}$, the transfer power is intensely larger, but at this time the efficiency is less than $50 \%$. When the axial distance is less than $5 \mathrm{~cm}$, the power and efficiency of the load reach a higher value simultaneously. Accordingly, the TL-RS mode is suitable for near distance transfer of less than $5 \mathrm{~cm}$.

In the $\mathrm{T}=\mathrm{R}$ mode, the transfer efficiency decreases monotonically with the growing of the axial and radial offset distance. At the axial offset $10 \mathrm{~cm}-14 \mathrm{~cm}$, the transfer power is intensely larger, but the efficiency is just about $50 \%$.

In the TS-RL mode, when the axial distance is $0 \mathrm{~cm}$ and the radial distance is $5 \mathrm{~cm}$, the transfer efficiency has the maximum value, and with the increase of the axial and radial migration distances, the efficiency decreases. If there is no radial offset at axial $12 \mathrm{~cm}-16 \mathrm{~cm}$, the transfer power is the most, and the efficiency is about 40\%-50\%. Moreover, the transfer power and efficiency have little influence by radial offset and maintain good transfer performance. When the axial $0 \mathrm{~cm}-10 \mathrm{~cm}$ and the large radial offset, both the transfer power and the efficiency can be achieved better.

Consequently, compared with TL-RS and T=R model, the TS-RL mode is suitable for axial long distance or larger radial offset at axial near distance transmission.

\section{Experiments}

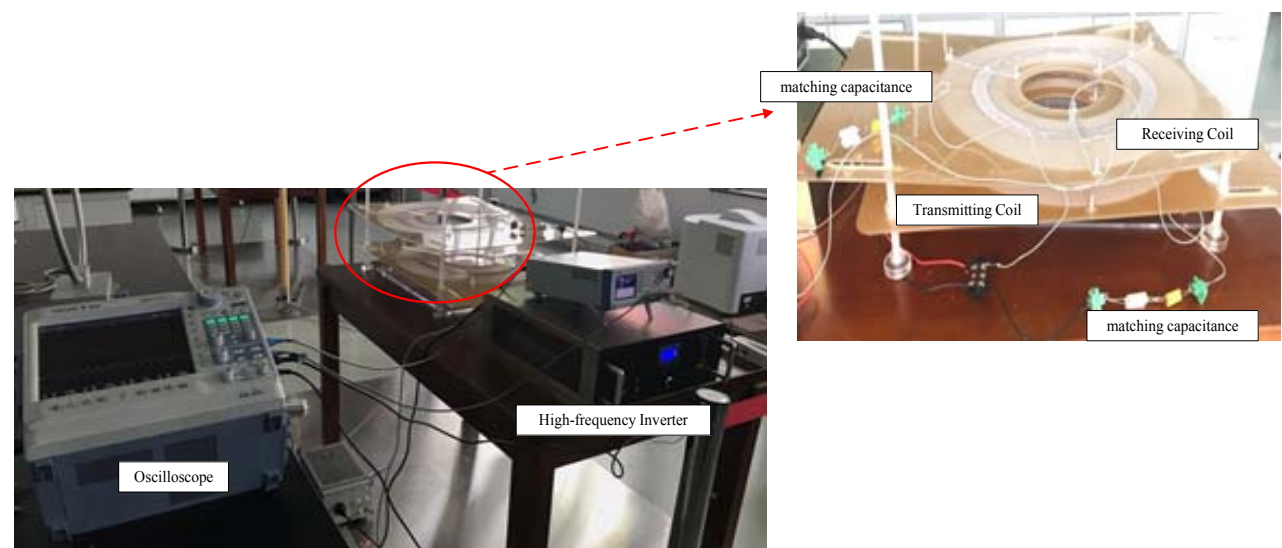

Fig.6 The test platform of MCRWPT system

In order to verify the above conclusion, a test platform of MCRWPT system is set up, as shown in Fig.6. The load is two $60 \mathrm{~W}$ bulbs with a resonant frequency of $150 \mathrm{kHz}$. The changing of transfer power and efficiency with the horizontal shift at different axial position are shown in the following Fig.7, where the $Z$ represents axial direction. The results show that when $Z$ is greater than $15 \mathrm{~cm}$ or less than $10 \mathrm{~cm}$ but greater radial offset, the TS-RL model get better power and efficiency than $\mathrm{T}=\mathrm{R}$ model.

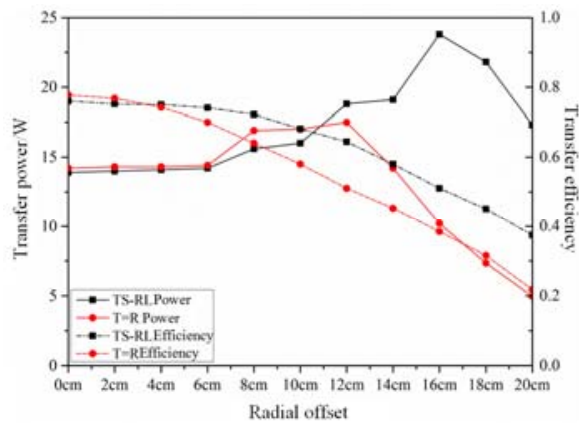

a) $Z=5 \mathrm{~cm}$

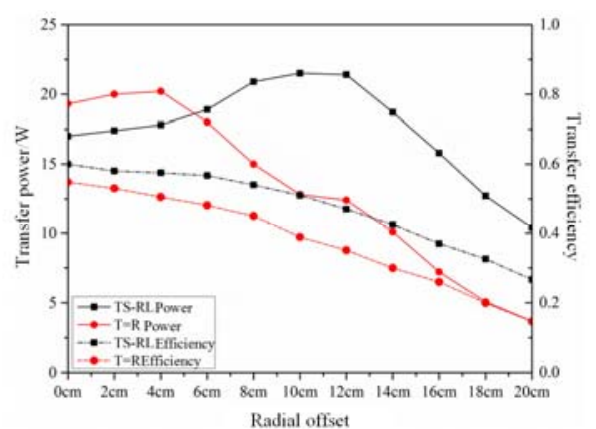

b) $\mathrm{Z}=10 \mathrm{~cm}$ 


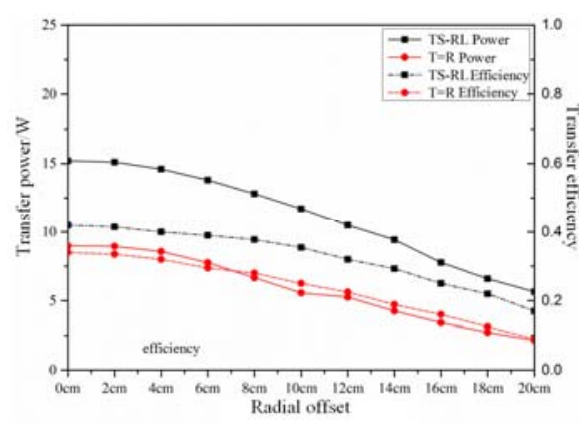

c) $\mathrm{Z}=15 \mathrm{~cm}$

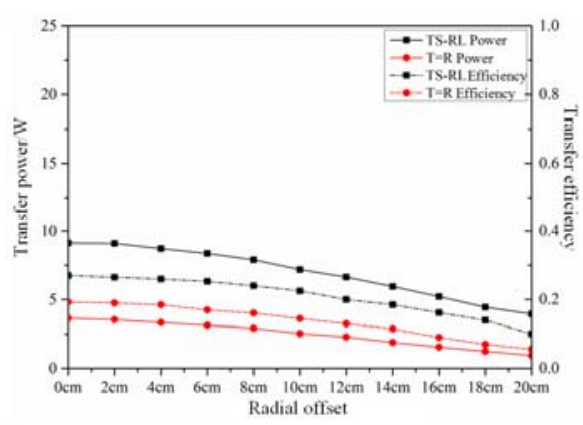

d) $Z=20 \mathrm{~cm}$

Fig.7 Load power and efficiency vary with the horizontal shift at different axial position

Based to the above simulation and experimental results, in order to adapt to the long-distance transmission and reduce the influence of the offset on the transfer efficiency, a optimization method by increasing the size of the receiving coil to get larger mutual inductance of the coils is proposed. The flow chart of efficiency optimization algorithm in the offset state is shown in Fig.7. Among it, $\mathrm{M}^{*}$ is the mutual inductance, $\mathrm{SP}^{*}$ is the size of the receiving coil and $\eta^{*}$ is the transfer efficiency in the initial state. Considering the actual situation of the coil, the mutual inductance of the coil can not be completely restored to the non offset state. Therefore, the aim to optimize the transfer efficiency is realized, when mutual inductance is maintained over $90 \%$ of the mutual inductance in the non offset state. In the experiment, the transmitting coil radius is fixed as $20 \mathrm{~cm}$, the receiving coil radius can be changed in the range of $15 \mathrm{~cm}-25 \mathrm{~cm}$. When the radial offset is $10 \mathrm{~cm}$, the axial offset is $10 \mathrm{~cm}$, using TS-RL mode can get the reduction efficiency rate decreased from $23.1 \%$ to $8.3 \%$.

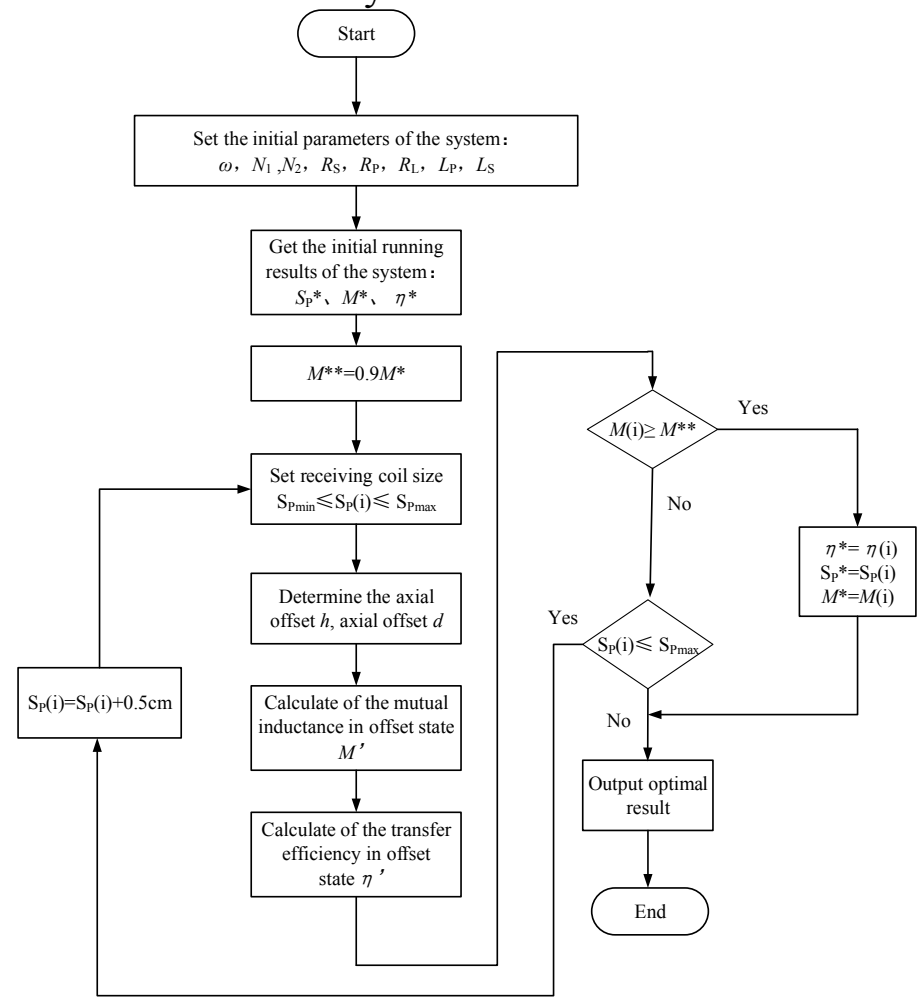

Fig.8 Flow chart of efficiency optimization algorithm in the offset state

\section{Conclusion}

In this paper, the parameters of mutual inductance, transfer power and efficiency under different modes are analyzed. The TS-RL transfer mode is more suitable for long distance transmission or near transmission with radial offset. And the transfer efficiency gets better by increasing the size of the receiving coil in the offset state. The effectiveness of the proposed optimization method has been proved through simulation and experimental results. 


\section{References}

[1]. FAN Xing-ming, MO Xiao-yong, ZHANG Xin. Research status and application of magnetic coupling resonant radio energy transmission. Transactions of China Electrotechnical Society[J], Vol. 28 (2013) No.12, p.75-82.

[2]. LI Yang, YANG Qing xin, YAN Zhuo, Analysis of the effective transmission distance of wireless energy and its influencing factors. Transactions of China Electrotechnical Society[J], Vol. 28 (2013) No.1, p.106-112.

[3]. XIAO Siyu, MA Dianguang, ZHANG Hanhua, Coil optimization of coupled resonant wireless power transmission system. Transactions of China Electrotechnical Society[J], Vol. 30 (2015) No.s1, p.221-225.

[4]. DU Xiu, WANG Jianqiang, CHENG Tengtian. Comparison and analysis results of two kinds of modeling for magnetically coupled wireless Power Transfer. Transactions of China Electrotechnical Society[J], Vol.28(2013) No.s2, p.7-12.

[5]. ZHAI Yuan, SUN Yue, Dai Xin, et al. Modeling and analysis of magnetic resonance wireless power transmission systems. Proceedings of the CSEE[J], Vol.32(2016) No.12, p.155-160.

[6]. YAN Maoshui, LIAO Chenglin, TAO Chengxuan, et al. A review on wireless power transfer coil misalignment: analysis, efficiency improvement. Transactions of China Electrotechnical Society[J], Vol.28(2013) No.s2, p.1-6. 